\title{
Coherently driven degenerate three-level laser with parametric amplifier
}

\author{
Tewodros Y Darge ${ }^{*}$, Fesseha Kassahun
}

\author{
* Correspondence: tewyirga@gmail. \\ com \\ Department of Physics, Addis \\ Ababa University, PO Box 33761, \\ Addis Ababa, Ethiopia
}

\begin{abstract}
We discuss the squeezing and statistical properties of the light produced by a coherently driven degenerate three-level laser with a parametric amplifier. We consider the case in which the atoms injected into the cavity are prepared in a coherent superposition of the top and bottom levels and with these levels coupled by the pump mode emerging from the parametric amplifier. It so happens that the presence of the parametric amplifier increases the squeezing and the mean photon number significantly. Furthermore, it is found that the maximum interacavity squeezing is $93 \%$ in the presence of the coupling and when the superposition has no contribution ( $\eta$ $=0$ ). On the other hand, the maximum interacavity squeezing turns out to be $94 \%$ in the absence of the coupling. This squeezing is due to the parametric amplifier and the superposition. In addition, our calculation shows that one effect of coupling the top and bottom levels is to decrease the mean and the normally-ordered variance of the photon number.
\end{abstract}

PACS codes: 42.55.Ah, 42.50.Lc, 42.50.Ar

\section{Introduction}

It has been established that a three-level laser under certain conditions generates squeezed light [1-9]. In a cascade three-level laser, three-level atoms in a cascade configuration are injected into a cavity coupled to a vacuum reservoir via a single-port mirror. The injected atoms may initially be prepared in a coherent superposition of the top and bottom levels and/or these levels may be coupled by strong coherent light after they are injected into the cavity. The superposition or the coupling of the top and bottom levels is responsible for the interesting nonclassical features of the generated light. When a three-level atom in a cascade configuration makes a transition from the top to the bottom level via the intermediate level, two photons are generated. If the two photons have the same frequency, the three-level atom is called degenerate otherwise it is called nondegenerate.

Some authors have studied the squeezing and statistical properties of the light produced by a three-level laser in which the crucial role is played by the superposition of the top and bottom levels [1-7]. Ansari [7] has predicted that such a laser can generate under certain conditions squeezed light. Furthermore, Lu and Zhu [2] have considered a nondegenerate three-level laser with the atoms initially prepared in coherent superposition of the top and bottom levels. They have predicted a maximum of $50 \%$ interacavity two-mode squeezing.

(c) 2010 Darge and Kassahun This is an open access article distributed under the terms of the Creative Commons Attribution License (http://creativecommons.org/licenses/by/2.0), which permits unrestricted use, distribution, and reproduction in any medium, provided the original work is properly cited. 
A three-level laser in which the top and bottom levels of the atoms injected into the cavity are coupled by a strong light has also been studied by different authors [7-9]. Ansari et al [9] have considered a degenerate three-level laser, with the atoms initially in the upper level and with the top and bottom levels of the atoms coupled by coherent light. They have shown that this system behaves like a parametric oscillator for sufficiently strong coherent light. They have also predicted that such a system can generate squeezed light over large range of the amplitude of the coherent light.

Furthermore, it has been predicted theoretically [10-16] and subsequently confirmed experimentally $[17,18]$ that a parametric oscillator produces light with a maximum interacavity squeezing of $50 \%$ below the coherent-state level. Some authors $[19,20]$ have considered a three-level laser whose cavity contains a parametric amplifier. Fesseha [19] has studied a three-level laser whose cavity contains a degenerate parametric amplifier, and with the injected atoms prepared initially in coherent superposition of the top and bottom levels. He has shown that the effect of the parametric amplifier is to increase the interacavity squeezing by a maximum of $50 \%$. He has also pointed out that since the presence of the parametric amplifier also leads to a significant increase in the mean photon number, the system can produce a bright and highly squeezed light. Moreover, Alebachew and Fesseha [20] have considered a degenerate three-level laser whose cavity contains a parametric amplifier, with the top and bottom levels of the injected atoms coupled by the pump mode emerging from the parametric amplifier. They have studied this system for the specific case in which the number of atoms initially in the top and bottom levels are equal. They have found that this system generates under certain conditions a highly squeezed light. The squeezing in this case is exclusively due to the parametric amplifier and the coupling of the top and bottom levels.

In this paper we seek to study a degenerate three-level laser whose cavity contains a parametric amplifier and with the cavity mode driven by a strong coherent light as shown in Figure 1. Moreover, the three-level atoms injected into the cavity are initially prepared in a coherent superposition of the top and bottom levels and with these levels coupled by the pump mode emerging from the parametric amplifier. In order to determine the squeezing and statistical properties of the light produced by this quantum optical system, we first derive c-number Langevin equations using the pertinent master equation. Employing the solutions of the resulting c-number Langevin equations along

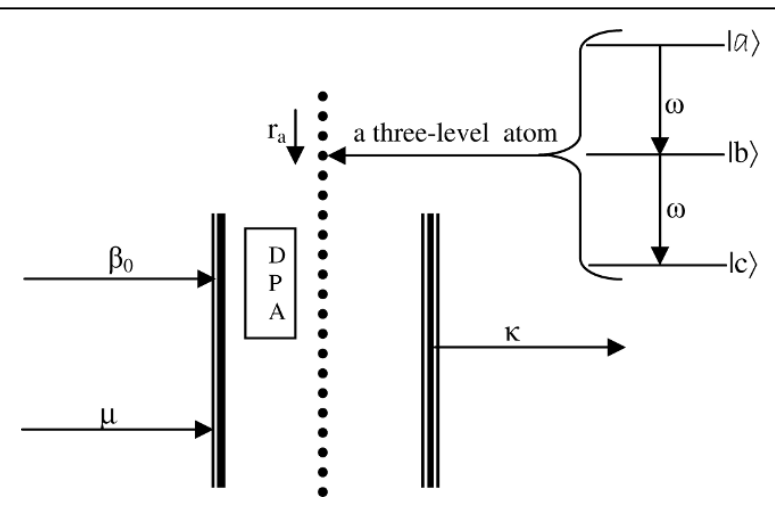

Figure 1 The system under consideration. A coherently driven degenerate three-level laser with a parametric amplifier. 
with the properties of the noise forces, we calculate the quadrature variance of the cavity and output modes. Applying the same solutions, we also obtain the antinormally ordered characteristic function with the aid of which the $Q$ function is determined. The resulting $Q$ function is then used to calculate the mean and the normally-ordered variance of the photon number as well as the photon number distribution for the cavity mode.

\section{2 c-number Langevin Equations}

Three-level atoms in a cascade configuration are injected at a constant rate into the laser cavity. We denote the top, middle, and bottom levels of a three-level atom by $|a\rangle$, $|b\rangle$, and $|c\rangle$, respectively. We assume the transitions $|a\rangle \rightarrow|b\rangle$ and $|b\rangle \rightarrow|c\rangle$ to be dipole allowed, with the transition $|a\rangle \rightarrow|c\rangle$ to be dipole forbidden. We consider the case for which the cavity mode is at resonance with the two transitions $|a\rangle \rightarrow|b\rangle$ and $|b\rangle \rightarrow|c\rangle$. We take the initial state of a three-level atom to be

$$
\left|\psi_{A}(0)\right\rangle=c_{a}(0)|a\rangle+c_{c}(0)|c\rangle .
$$

The initial density operator for the three-level atom can then be written as

$$
\hat{\rho}_{A}(0)=\rho_{a a}^{(0)}|a\rangle\left\langle a\left|+\rho_{a c}^{(0)}\right| a\right\rangle\left\langle c\left|+\rho_{c a}^{(0)}\right| c\right\rangle\left\langle a\left|+\rho_{c c}^{(0)}\right| c\right\rangle\langle c|,
$$

in which $\rho_{a a}^{(0)}=\left|c_{a}\right|^{2}, \rho_{a c}^{(0)}=c_{a} c_{c}^{*}, \rho_{c a}^{(0)}=c_{c} c_{a}^{*}, \rho_{c c}^{(0)}=\left|c_{c}\right|^{2}$. It proves to be convenient to introduce a new parameter $\eta$ defined by [7]

$$
\rho_{a a}^{(0)}=\frac{1-\eta}{2}
$$

Using the fact that

$$
\rho_{a a}^{(0)}+\rho_{c c}^{(0)}=1
$$

along with

$$
\left|\rho_{a c}^{(0)}\right|^{2}=\rho_{a a}^{(0)} \rho_{c c}^{(0)},
$$

one easily finds

$$
\rho_{c c}^{(0)}=\frac{1+\eta}{2}
$$

and

$$
\left|\rho_{a c}^{(0)}\right|=\frac{1}{2} \sqrt{1-\eta^{2}} .
$$

We note that the parameter $\eta$ describes the coherent superposition of the top and bottom levels at the initial time. Upon setting

$$
\rho_{a c}^{(0)}=\left|\rho_{a c}^{(0)}\right| e^{i \theta},
$$


expression (2) can be put in the form

$$
\hat{\rho}_{A}(0)=\frac{1-\eta}{2}|a\rangle\left\langle a\left|+\frac{1}{2} \sqrt{1-\eta^{2}} e^{i \theta}\right| a\right\rangle\left\langle c\left|+\frac{1}{2} \sqrt{1-\eta^{2}} e^{-i \theta}\right| c\right\rangle\left\langle a\left|+\frac{1+\eta}{2}\right| c\right\rangle\langle c| .
$$

The interaction of a three-level atom with the cavity mode and the pump mode emerging from the parametric amplifier, the parametric down-conversion, and the interaction of the driving light with the cavity mode can be described by the Hamiltonian

$$
\begin{aligned}
\hat{H} & =i g\left(\hat{a}^{\dagger}(|b\rangle\langle a|+| c\rangle\langle b|)-\hat{a}(|a\rangle\langle b|+| b\rangle\langle c|)\right) \\
& +\frac{i \Omega}{2}(|c\rangle\langle a|-| a\rangle\langle c|)+\frac{i \varepsilon}{2}\left(\hat{a}^{\dagger 2}-\hat{a}^{2}\right)+i \mu\left(\hat{a}^{\dagger}-\hat{a}\right),
\end{aligned}
$$

in which $\hat{a}$ is the annihilation operator for the cavity mode, $g$ is the atom-cavity mode coupling constant (assumed to be the same for all the three-levels), $\Omega=2 \lambda \beta_{0}$ (with $\beta_{0}$ and $\lambda$ being the amplitude of the pump mode and the atom-pump mode coupling constant), $\varepsilon=\lambda^{\prime} \beta_{0}$ (with $\lambda^{\prime}$ being the coupling constant between the pump mode and the signal mode), and $\mu$ is proportional to the amplitude of the driving light. Now taking into account the interaction of the cavity mode with the vacuum reservoir and for $\theta=0$, the master equation for the cavity mode of the quantum optical system under consideration is found in the linear and adiabatic approximation schemes to be [3]

$$
\begin{aligned}
\frac{d \hat{\rho}}{d t}= & \mu\left(\hat{a}^{\dagger} \hat{\rho}-\hat{\rho} \hat{a}^{\dagger}+\hat{p} \hat{a}-\hat{a} \hat{\rho}\right)+\frac{\varepsilon}{2}\left(\hat{\rho} \hat{a}^{2}-\hat{a}^{2} \hat{\rho}+\hat{a}^{\dagger 2} \hat{\rho}-\hat{\rho} \hat{a}^{\dagger 2}\right) \\
& +R\left(2 \hat{a}^{\dagger} \hat{\rho} \hat{a}-\hat{a} \hat{a}^{\dagger} \hat{\rho}-\hat{\rho} \hat{a} \hat{a}^{\dagger}\right)+S\left(2 \hat{a} \hat{\rho} \hat{a}^{\dagger}-\hat{\rho} \hat{a}^{\dagger} \hat{a}-\hat{a}^{\dagger} \hat{a} \hat{\rho}\right) \\
& +U\left(\hat{a}^{\dagger} \hat{\rho} \hat{a}^{\dagger}+\hat{a} \hat{\rho} \hat{a}-\hat{a}^{2} \hat{\rho}-\hat{\rho} \hat{a}^{\dagger 2}\right)+V\left(\hat{a}^{\dagger} \hat{\rho} \hat{a}^{\dagger}+\hat{a} \hat{\rho} \hat{a}-\hat{\rho} \hat{a}^{2}-\hat{a}^{\dagger 2} \hat{\rho}\right),
\end{aligned}
$$

where

$$
\begin{aligned}
& R=\frac{A}{4 B}\left(\left(\frac{\beta^{2}}{2}-1\right) \eta-\frac{3}{2} \beta \sqrt{1-\eta^{2}}+\left(1+\beta^{2}\right)\right) \\
& S=\frac{A}{4 B}\left(\frac{2 \kappa B}{A}+\left(1-\frac{\beta^{2}}{2}\right) \eta+\frac{3}{2} \beta \sqrt{1-\eta^{2}}+\left(1+\beta^{2}\right)\right) \\
& U=\frac{A}{4 B}\left(\frac{3}{2} \beta \eta+\left(\frac{\beta^{2}}{2}-1\right) \sqrt{1-\eta^{2}}+\left(\frac{\beta}{2}+\frac{\beta^{3}}{2}\right)\right) \\
& V=\frac{A}{4 B}\left(\frac{3}{2} \beta \eta+\left(\frac{\beta^{2}}{2}-1\right) \sqrt{1-\eta^{2}}-\left(\frac{\beta}{2}+\frac{\beta^{3}}{2}\right)\right)
\end{aligned}
$$

with

$$
\beta=\frac{\Omega}{\gamma}
$$




$$
B=\left(1+\beta^{2} / 4\right)\left(1+\beta^{2}\right)
$$

and

$$
A=\frac{2 r_{a} g^{2}}{\gamma^{2}} .
$$

Here $\gamma$, considered to be the same for all the three-levels, is the atomic decay constant and $A$ is the linear gain coefficient.

Now employing the relation

$$
\frac{d}{d t}\left\langle z\left(\hat{a}, \hat{a}^{\dagger}\right)\right\rangle=\operatorname{Tr}\left(\frac{d \hat{\rho}}{d t} z\left(\hat{a}, \hat{a}^{\dagger}\right)\right)
$$

along with the master equation (11), one readily finds the following equations:

$$
\begin{aligned}
\frac{d}{d t}\langle\hat{a}(t)\rangle= & (R-S)\langle\hat{a}(t)\rangle+(U-V+\varepsilon)\left(\hat{a}^{\dagger}(t)\right\rangle+\mu, \\
\frac{d}{d t}\left\langle\hat{a}^{2}(t)\right\rangle= & 2(R-S)\left\langle\hat{a}^{2}(t)\right\rangle+2(U-V+\varepsilon)\left\langle\hat{a}^{\dagger}(t) \hat{a}(t)\right\rangle \\
& +2 \mu\langle\hat{a}(t)\rangle+(\varepsilon-2 V),
\end{aligned}
$$

and

$$
\begin{aligned}
& \frac{d}{d t}\left\langle\hat{a}^{\dagger}(t) \hat{a}(t)\right\rangle=2(R-S)\left\langle\hat{a}^{\dagger}(t) \hat{a}(t)\right\rangle+(U-V+\varepsilon)\left(\left\langle\hat{a}^{\dagger 2}(t)\right\rangle\right. \\
& \left.+\left\langle\hat{a}^{2}(t)\right\rangle\right)+\mu\left(\left\langle\hat{a}^{\dagger}(t)\right\rangle+\langle\hat{a}(t)\rangle\right)+2 R .
\end{aligned}
$$

We note that the operators in the above set of equations are in the normal order. Thus the c-number equations associated with this ordering are

$$
\begin{gathered}
\frac{d}{d t}\langle\alpha(t)\rangle=-(S-R)\langle\alpha(t)\rangle+(U-V+\varepsilon)\left\langle\alpha^{*}(t)\right\rangle+\mu, \\
\frac{d}{d t}\left\langle\alpha^{2}(t)\right\rangle=-2(S-R)\left\langle\alpha^{2}(t)\right\rangle+2(U-V+\varepsilon)\left\langle\alpha^{*}(t) \alpha(t)\right\rangle+2 \mu\langle\alpha(t)\rangle+(\varepsilon-2 V),
\end{gathered}
$$

and

$$
\left.\frac{d}{d t}\left\langle{ }^{\prime \prime}(t)(t)\right\rangle=-2(S-R)\left\langle{ }^{*}(t)(t)\right\rangle+(U-V+) \ll\left({ }^{22}(t)\right\rangle+\left\langle{ }^{2}(t)\right\rangle\right)+(\langle "(t)\rangle+\langle(t)\rangle)+2 R .
$$

We claim that the equation of evolution of $\alpha(t)$ (c-number Langevin equation) can be obtained from that of $\langle\alpha(t)\rangle$. This can be achieved by dropping the angular brackets in Eq. (23) and adding a noise force $f(t)$, so that

$$
\frac{d}{d t} \alpha(t)=-(S-R) \alpha(t)+(U-V+\varepsilon) \alpha^{*}(t)+\mu+f(t) .
$$


With the aid of Eqs. (23), (24), (25), and (26), one can readily establish the correlation properties of the noise force [3]

$$
\langle f(t)\rangle=0,
$$

$$
\left\langle f(t) f\left(t^{\prime}\right)\right\rangle=(\varepsilon-2 V) \delta\left(t-t^{\prime}\right),
$$

and

$$
\left\langle f^{*}(t) f\left(t^{\prime}\right)\right\rangle=\left\langle f(t) f^{*}\left(t^{\prime}\right)\right\rangle=2 R \delta\left(t-t^{\prime}\right) .
$$

Now introducing a new variable defined by

$$
\alpha_{ \pm}(t)=\alpha^{*}(t) \pm \alpha(t)
$$

one can easily show using (26) and its complex conjugate that

$$
\frac{d}{d t} \alpha_{ \pm}(t)=-\lambda_{\mp} \alpha_{ \pm}(t)+(\mu \pm \mu)+f^{*}(t) \pm f(t),
$$

where

$$
\lambda_{\mp}=(S-R) \mp(U-V+\varepsilon) .
$$

The solution of (31) can be put in the form

$$
\alpha_{ \pm}(t)=\alpha_{ \pm}(0) e^{-\lambda_{\mp} t}+\int_{0}^{t} e^{-\lambda_{\mp}\left(t-t^{\prime}\right)}\left[\mu \pm \mu+f^{*}\left(t^{\prime}\right) \pm f\left(t^{\prime}\right)\right] d t^{\prime} .
$$

so that in view of (30), there follows

$$
\alpha(t)=B_{+}(t) \alpha(0)+B_{-}(t) \alpha^{*}(0)+E(t)+F(t),
$$

in which

$$
\begin{aligned}
& B_{ \pm}(t)=\frac{1}{2}\left(e^{-\lambda_{-} t} \pm e^{-\lambda_{+} t}\right), \\
& E(t)=\frac{\mu}{\lambda_{-}}\left(1-e^{-\lambda_{-} t}\right),
\end{aligned}
$$

and

$$
F(t)=F_{+}(t)+F_{-}(t),
$$

with

$$
F_{ \pm}=\frac{1}{2} \int_{0}^{t} e^{-\lambda_{\mp}\left(t-t^{\prime}\right)}\left(f\left(t^{\prime}\right) \pm f^{*}\left(t^{\prime}\right)\right) d t^{\prime} .
$$




\section{Quadrature Squeezing of the Cavity Mode}

The squeezing properties of a single-mode light are described by two quadrature operators

$$
\hat{a}_{+}=\hat{a}+\hat{a}^{\dagger}
$$

and

$$
\hat{a}_{-}=i\left(\hat{a}^{\dagger}-\hat{a}\right) .
$$

The quadrature variance, defined by

$$
\Delta a_{ \pm}^{2}(t)=\left\langle a_{ \pm}^{2}(t)\right\rangle-\left\langle a_{ \pm}(t)\right\rangle^{2}
$$

can be expressed in terms of c-number variables associated with the normal ordering as

$$
\Delta a_{ \pm}^{2}(t)=1 \pm\left\langle\alpha_{ \pm}(t), \alpha_{ \pm}(t)\right\rangle
$$

Employing (33) along with (27), (28), and (29), we obtain at steady state

$$
\left\langle\alpha_{ \pm}(t)\right\rangle_{s s}=\frac{\mu \pm \mu}{\lambda_{\mp}}
$$

and

$$
\left\langle\alpha_{ \pm}^{2}(t)\right\rangle_{s s}=\frac{(\varepsilon-2 V \pm 2 R)}{\lambda_{\mp}}+\frac{(\mu \pm \mu)^{2}}{\lambda_{\mp}^{2}} .
$$

Hence substitution of these results into (42) yields

$$
\Delta a_{ \pm s s}^{2}=1 \pm \frac{\varepsilon-2 V \pm 2 R}{\lambda \mp} .
$$

Furthermore, on account of (12)-(15), expression (32) takes the form

$$
\lambda_{\mp}=\frac{\kappa}{2}+\frac{3 A \beta \sqrt{1-\eta^{2}}+A\left(2-\beta^{2}\right) \eta \mp A\left(\beta+\beta^{3}\right)}{4\left(1+\beta^{2}\right)\left(1+\beta^{2} / 4\right)} \mp \varepsilon .
$$

Finally, application of (12), (15), and (46) in (45) leads to

$$
\Delta a_{ \pm}^{2}=\frac{2 \kappa\left(1+\beta^{2}\right)\left(1+\beta^{2} / 4\right) \pm A\left(2-\beta^{2}\right) \sqrt{1-\eta^{2}}+2 A\left(1+\beta^{2}\right) \mp 3 A \beta \eta}{(2 \kappa \mp 4 \varepsilon)\left(1+\beta^{2}\right)\left(1+\beta^{2} / 4\right)+3 A \beta \sqrt{1-\eta^{2}} \mp A\left(\beta+\beta^{3}\right)+A\left(2-\beta^{2}\right) \eta} .
$$

We see from (47) that the driving light has no effect on the quadrature variance. Now inspection of (46) shows that $\lambda_{+}$is nonnegative while $\lambda_{-}$can be positive, negative, or zero. It turns out to be useful to write (46) as

$$
\lambda_{-}=G-\varepsilon,
$$


with

$$
G=\frac{\kappa}{2}+\frac{3 A \beta \sqrt{1-\eta^{2}}+A(2-\beta) \eta-A\left(\beta+\beta^{3}\right)}{4\left(1+\beta^{2}\right)\left(1+\beta^{2} / 4\right)} .
$$

We observe that the equation of evolution of $\alpha_{-}(t)$, described by (31), does not have a well-behaved solution for $\varepsilon>G$. We then identify $\varepsilon=G$ as the threshold condition.

We next proceed to analyze the quadrature variance of the light generated by the system operating below threshold. Using (47) and (49) and writing a simple Matlab program, we have obtained for $A=100$ and $\kappa=0.8$ the values of $\eta, \beta$, $\varepsilon$, and $\Delta a_{-}^{2}$ for which (31) has a solution. It so turns out that this equation has a solution for $-0.5 \leq \eta \leq 1$ and for $0 \leq \beta \leq$ 1.4. We indicate in Table 1 the values of $\eta, \beta$, and $\varepsilon$ corresponding to the two smallest values of the quadrature variance. We note that when there are equal number of atoms initially in the top and bottom levels $(\eta=0)$, the maximum interacavity squeezing is $93 \%$ below the coherent-state level for $\beta=0.1$. On the other hand, when there are slightly more atoms initially in the bottom level than in the top level $(\eta=0.1)$, the maximum interacavity squeezing is found to be $94 \%$ below the coherent-state level for $\beta=0$.

It is interesting to examine some special cases. First we consider the case in which the nonlinear crystal is removed from the cavity, with the top and bottom levels of the atoms coupled by the pump mode. Thus upon setting $\varepsilon=0$ (with $\beta_{0} \neq 0$ ) in Eq. (47), we get

$$
\Delta a_{ \pm}^{2}=\frac{2 \kappa\left(1+\beta^{2}\right)\left(1+\beta^{2} / 4\right) \pm A\left(2-\beta^{2}\right) \sqrt{1-\eta^{2}}+2 A\left(1+\beta^{2}\right) \mp 3 A \beta \eta}{2 \kappa\left(1+\beta^{2}\right)\left(1+\beta^{2} / 4\right)+3 A \beta \sqrt{1-\eta^{2}} \mp A\left(\beta+\beta^{3}\right)+A\left(2-\beta^{2}\right) \eta} .
$$

It can be shown using (50) that for $\eta=0, \beta=0$, and any values of $A$ and $\kappa$ the light generated is not in a squeezed state. However, for $\eta=0, A=100$, and $\kappa=0.8$ we readily get applying the same equation that the maximum squeezing to be $89 \%$ for $\beta=$ 0 . We therefore infer that the squeezing in this case is exclusively due to the coupling of the top and bottom levels. Moreover, for $\eta=0.1$ and for the above values of $A$ and $\kappa$, we find the maximum squeezing to be $88 \%$ for $\beta=0$. This squeezing is exclusively due to the superposition of the top and bottom levels. In addition, inspection of the plots in Figure 2 shows that for small values of the amplitude of the pump mode, the coupling of the top and bottom levels significantly enhances the intracavity squeezing particularly when there are equal number of atoms initially in the top and bottom levels $(\eta=0)$. Otherwise, it leads to the decrease in the intercavity squeezing. On the other hand, for a strong pump mode $(\beta \gg 1)$, Eq. (50) takes the form

$$
\Delta a_{ \pm}^{2}=\frac{\frac{\kappa}{2} \pm \frac{A}{\beta^{2}} \sqrt{1-\eta^{2}}+\frac{2 A}{\beta^{2}} \mp \frac{3 A}{\beta^{3}} \eta}{\frac{\kappa}{2}+\frac{3 A}{\beta^{2}} \sqrt{1-\eta^{2}} \mp \frac{A}{\beta}-\frac{A}{\beta^{2}} \eta}
$$

Table 1 Quadrature variance

\begin{tabular}{ccccc}
\hline \multicolumn{5}{c}{ Quadrature Variance } \\
\hline$\eta$ & $\beta$ & $G$ & $\varepsilon$ & $\Delta a_{-}^{2}$ \\
\hline 0 & 0.1000 & 5.3135 & 5.3000 & 0.0731 \\
\hline 0.1000 & 0 & 5.4000 & 5.3000 & 0.0608 \\
\hline
\end{tabular}

Values of $\Delta a_{-}^{2}$ for $A=100$ and $\kappa=0.8$. 


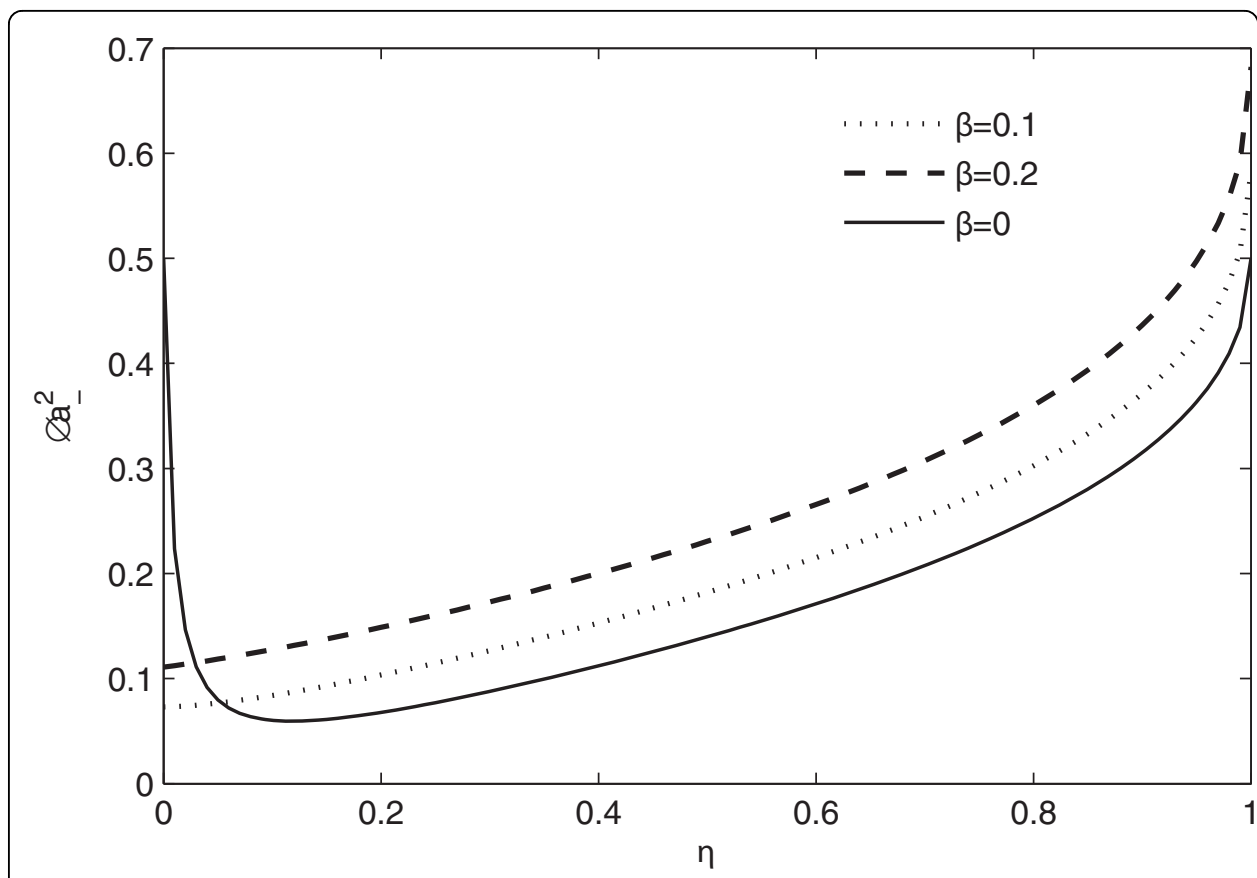

Figure 2 Effect of the coupling on the quadrature variance. Plots of the quadrature variance [Eq. (50)] versus $\eta$ for $A=100, \kappa=0.8$, and different values of $\beta$.

so that on dropping the terms $\frac{A}{\beta^{2}}$ and $\frac{A}{\beta^{3}}$, there follows

$$
\Delta a_{ \pm}^{2}=\frac{1}{1 \mp \frac{2 A}{\beta \kappa}} .
$$

This result indicates that a degenerate three-level laser driven by a strong light behaves like a degenerate parametric oscillator [9].

Furthermore, we consider the special case in which the pump mode emerging from the nonlinear crystal does not couple the top and bottom levels. Hence upon setting $\beta=0$ (with $\beta_{0} \neq 0$ ), Eq. (47) reduces to [19]

$$
\Delta a_{ \pm}^{2}=\frac{\kappa \pm A\left[1+(1-\eta)^{1 / 2}\right]}{A \eta+\kappa \mp 2 \varepsilon} .
$$

It is apparent that $\varepsilon$ is the only parameter representing the parametric amplifier. And inspection of Eq. (53) shows that one effect of the parametric amplifier is to decrease the value of the variance of the minus quadrature.

The plots in Figure 3 clearly indicate that the presence of the nonlinear crystal leads to better squeezing. In addition, applying Eq. (53) with $A=100, k=0.8$, and $\eta=0.1$ the maximum interacavity squeezing is $94 \%$ below the coherent-state level for $\varepsilon=5.3$.

\section{Quadrature Squeezing of the Output Mode}

Using the input-output relation, one can readily establish that the quadrature variance for the output mode is expressible as [3]

$$
\Delta a_{\text {out } \pm}^{2}=\kappa \Delta a_{ \pm}^{2}+(1-\kappa) \Delta a_{\text {in }}^{2}
$$




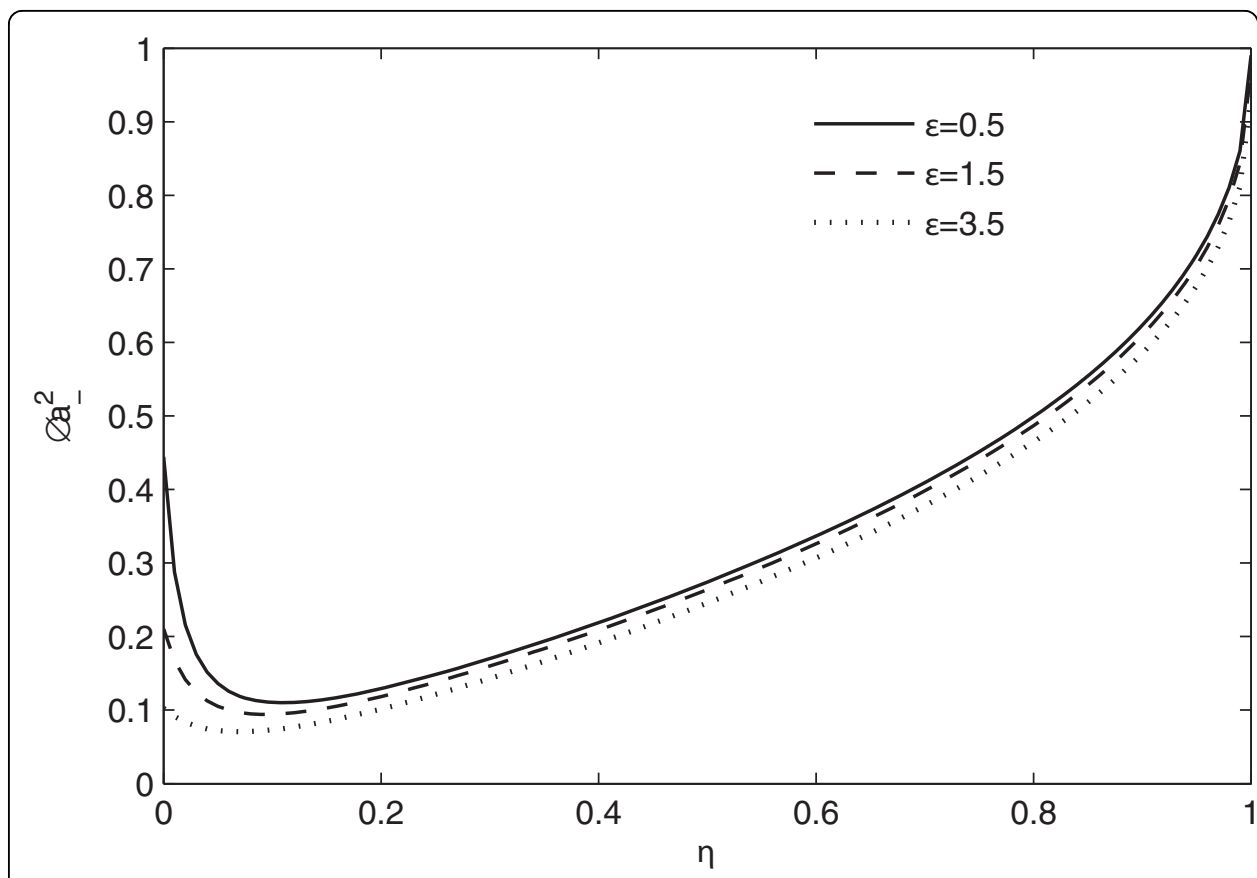

Figure 3 Effect of the parametric amplifier on the quadrature variance. Plots of the quadrature variance [Eq. (53)] versus $\eta$ for $A=100, \kappa=0.8$, and different values of $\varepsilon$.

where the first and the second terms represent the quadrature variance for the transmitted and reflected output mode. Taking into account (45) and the fact that the quadrature variance for the vacuum reservoir is unity, we get

$$
\Delta a_{\text {out } \pm}^{2}(t)=1 \pm \frac{\kappa(\varepsilon-2 V \pm 2 R)}{\lambda \mp} .
$$

It can be seen from the plots in Figure 4 that in general the cavity mode squeezing is greater than the output mode squeezing. Furthermore, applying Eq. (55) with $\eta=0.1$, $\varepsilon=5.3, A=100$, and $\kappa=0.8$, the maximum squeezing for the output mode is found to be $75 \%$ (occurs at $\beta=0$ ).

\section{Photon Statistics of the Cavity Mode}

In order to determine the photon statistics of the cavity mode, we first obtain the $Q$ function. The $Q$ function for a single-mode light can be written as

$$
Q\left(\alpha^{*}, \alpha, t\right)=\frac{1}{\pi^{2}} \int d z^{2} \phi(z, t) \exp \left(z^{*} \alpha-z \alpha^{*}\right),
$$

with the characteristic function $\varphi\left(z^{*}, z, t\right)$ defined in the Heisenberg picture by

$$
\phi\left(z^{*}, z, t\right)=\operatorname{Tr}\left(\rho(0) e^{-z^{*} a(t)} e^{z a(t)}\right) .
$$

This can be expressed in terms of c-number variables associated with the normal ordering as [3]

$$
\phi\left(z^{*}, z, t\right)=e^{-z^{*} z}\left\langle\exp \left(z \alpha^{*}-z^{*} \alpha\right)\right\rangle .
$$




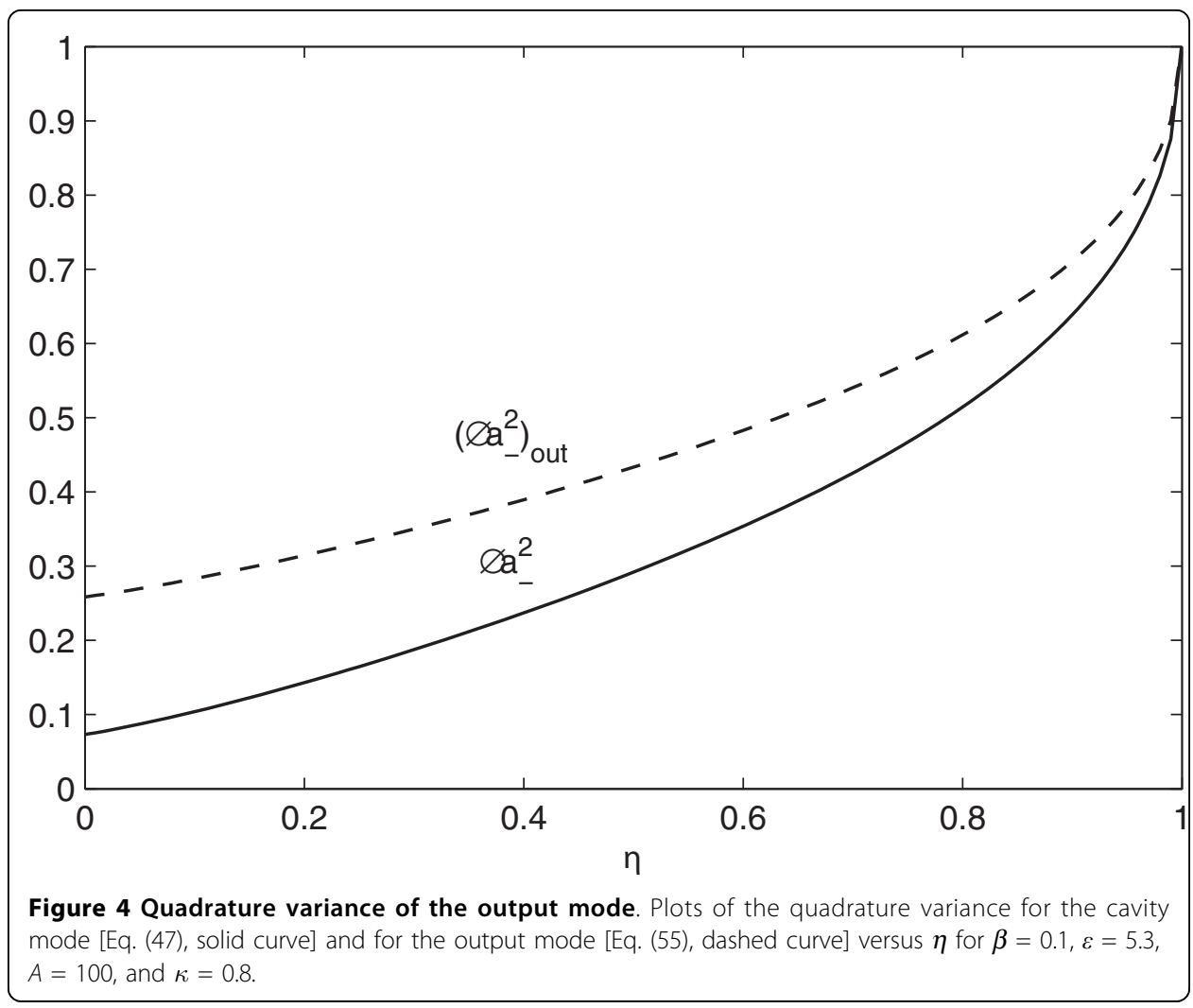

One can rewrite (34) as

$$
\alpha(t)=\alpha^{\prime}(t)+E(t)
$$

where

$$
\alpha^{\prime}(t)=B_{+}(t) \alpha(0)+B_{-}(t) \alpha^{*}(0)+F(t) .
$$

On account of (59) and its complex conjugate, we have

$$
\phi\left(z^{*}, z, t\right)=e^{-z^{*} z+\left(z-z^{*}\right) E}\left\langle\exp \left(z \alpha^{\prime *}-z^{*} \alpha^{\prime}\right)\right\rangle .
$$

Now with the aid of (60) along with (32), (35), (37), and (38), the equation of evolution of the expectation value of $\alpha^{\prime}(t)$ can be written as

$$
\frac{d}{d t}\left\langle\alpha^{\prime}(t)\right\rangle=-(S-R)\left\langle\alpha^{\prime}(t)\right\rangle+(U-V+\varepsilon)\left\langle\alpha^{\prime *}(t)\right\rangle .
$$

We see from this equation that $\alpha^{\prime}(t)$ is a Gaussian variable. In addition, on account of (60) along with the assumption that the cavity mode is initially in a vacuum state, we easily see that $\left\langle\alpha^{\prime}(t)\right\rangle=0$. Hence $\alpha^{\prime}(t)$ is a Gaussian variable with a vanishing mean. One can then express (61) in the form [3]

$$
\phi\left(z^{*}, z, t\right)=e^{-z z^{*}+\left(z-z^{*}\right) E} \exp \left(\frac{1}{2}\left\langle\left[z^{2} \alpha^{\prime * 2}+z^{* 2} \alpha^{\prime 2}-2 z z^{*} \alpha^{\prime} \alpha^{\prime *}\right]\right\rangle\right) .
$$


On the other hand, employing Eqs. (37), (38), and (60) along with the correlation properties of the noise force, one readily finds

$$
\left\langle\alpha^{\prime 2}(t)\right\rangle=\frac{2 R-2 V+\varepsilon}{4 \lambda_{-}}\left(1-e^{-2 \lambda_{-} t}\right)-\frac{2 R+2 V-\varepsilon}{4 \lambda_{+}}\left(1-e^{-2 \lambda_{+} t}\right)
$$

and

$$
\left\langle\alpha^{\prime *}(t) \alpha^{\prime}(t)\right\rangle=\frac{2 R-2 V+\varepsilon}{4 \lambda_{-}}\left(1-e^{-2 \lambda_{-} t}\right)+\frac{2 R+2 V-\varepsilon}{4 \lambda_{+}}\left(1-e^{-2 \lambda_{+} t}\right) .
$$

On substituting (64) and (65) into Eq. (63), there follows

$$
\phi\left(z^{*}, z, t\right)=\exp \left(-a z^{*} z-\frac{b}{2}\left(z^{2}+z^{*}\right)+\left(z-z^{*}\right) E\right)
$$

in which

$$
a=1+\frac{2 R-2 V+\varepsilon}{4 \lambda_{-}}\left(1-e^{-2 \lambda_{-} t}\right)+\frac{2 R+2 V-\varepsilon}{4 \lambda_{+}}\left(1-e^{-2 \lambda_{+} t}\right)
$$

and

$$
b=\frac{2 R-2 V+\varepsilon}{4 \lambda_{-}}\left(1-e^{-2 \lambda_{-} t}\right)-\frac{2 R+2 V-\varepsilon}{4 \lambda_{+}}\left(1-e^{-2 \lambda_{+} t}\right) .
$$

Now introducing (66) into Eq. (56) and carrying out the integration, we obtain

$$
\begin{aligned}
Q\left(\alpha^{*}, \alpha, t\right)= & \frac{\left(c^{2}-d^{2}\right)^{\frac{1}{2}}}{\pi} \exp \left[-c\left(\alpha^{*} \alpha-\alpha E-\alpha^{*} E+E^{2}\right)\right. \\
& \left.-\frac{d}{2}\left(\alpha^{2}+\alpha^{* 2}-2 E \alpha-2 E \alpha^{*}+2 E^{2}\right)\right]
\end{aligned}
$$

where

$$
c=\frac{a}{a^{2}-b^{2}}
$$

and

$$
d=\frac{b}{a^{2}-b^{2}} .
$$

\subsection{Mean and variance of the photon number}

The interacavity photon number is represented by the operator

$$
\hat{n}=\hat{a}^{\dagger} \hat{a}
$$


On the other hand, the expectation value of an operator function $\hat{A}\left(\hat{a}^{\dagger}, \hat{a}\right)$ can be expressed in terms of the $Q$ function as

$$
\langle\hat{A}\rangle=\int d \alpha^{2} Q(\alpha) A(\alpha),
$$

in which $A(\alpha)$ is the c-number function corresponding to the operator function $\hat{A}\left(\hat{a}^{\dagger}, \hat{a}\right)$ in the antinormal order. The mean of the photon number for the cavity mode can therefore be written as

$$
\bar{n}=\int d \alpha^{2} Q(\alpha)\left(\alpha^{*} \alpha-1\right)
$$

so that applying the $Q$ function (69) and carrying out the integration, we get

$$
\bar{n}=E^{2}+a-1 \text {. }
$$

On account of Eqs. (36) and (67), the mean of the photon number for the cavity mode is found at steady state to be

$$
\bar{n}_{s s}=\frac{\mu^{2}}{\lambda_{-}^{2}}+\frac{2 R-2 V+\varepsilon}{4 \lambda_{-}}+\frac{2 R+2 V-\varepsilon}{4 \lambda_{+}} .
$$

The plots in Figure 5 clearly indicate that the parametric amplifier and the driving light contribute significantly to the mean of the photon number. On the other hand, the plots in Figure 6 show that the mean of the photon number decreases with $\eta$. This

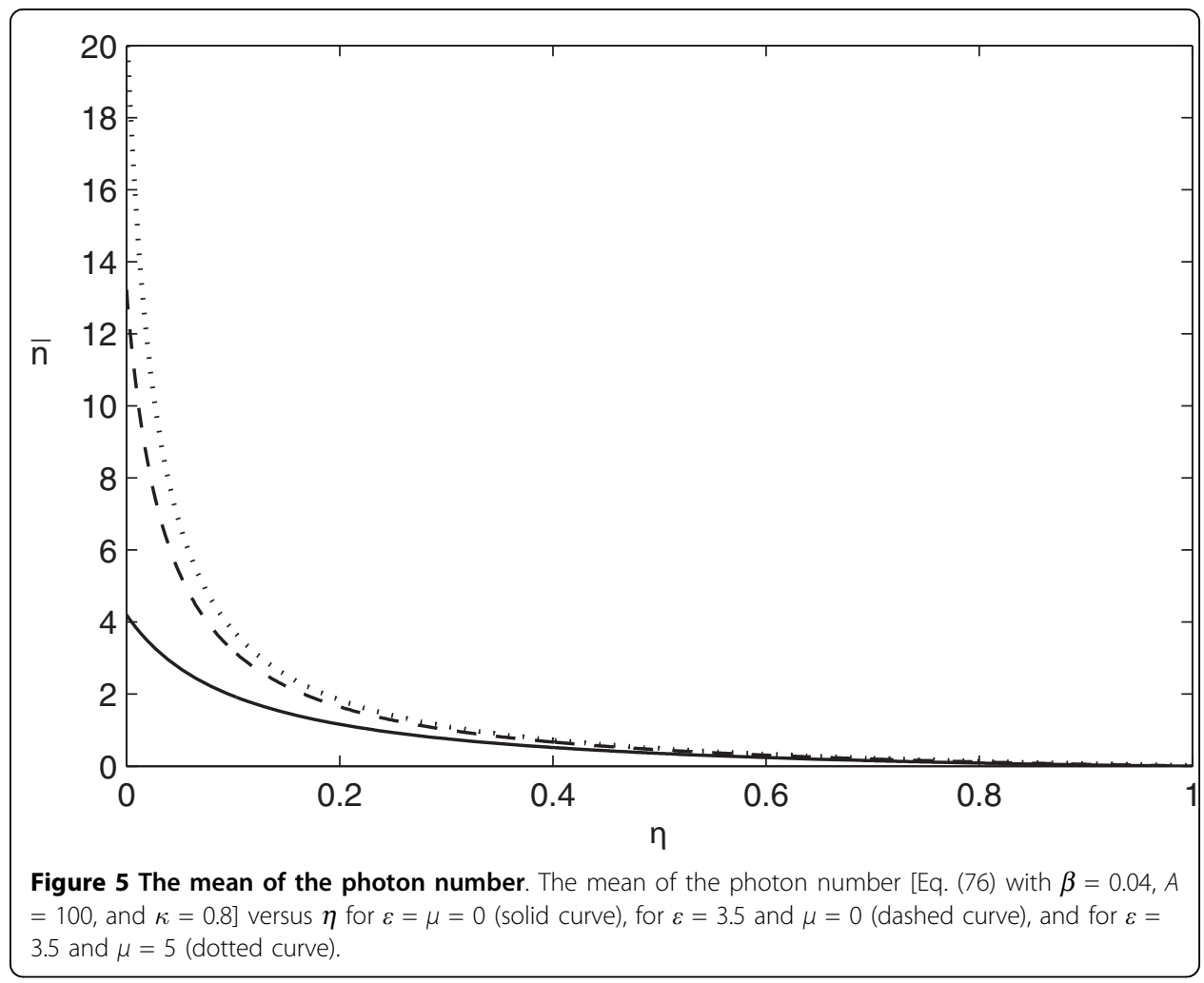




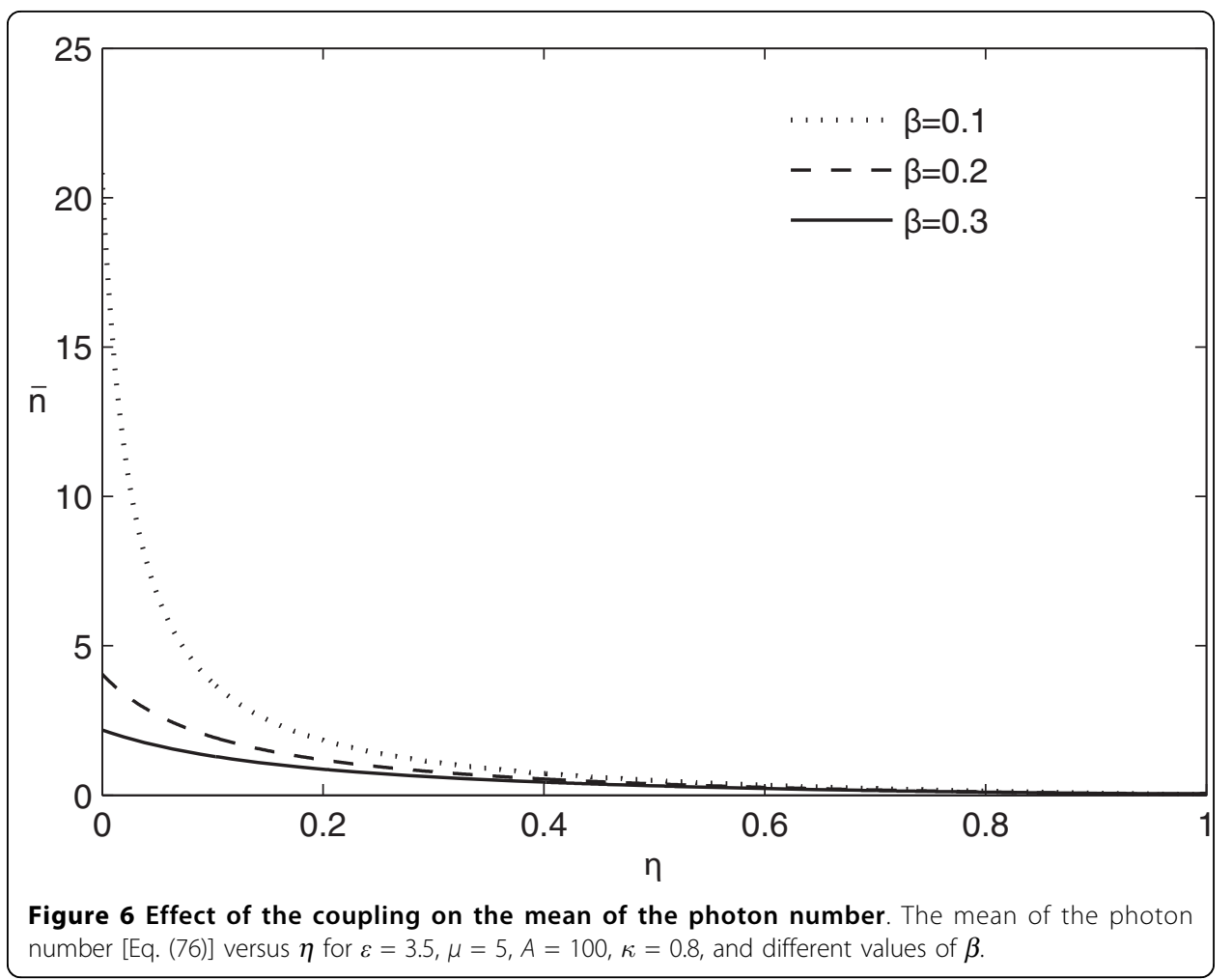

must be due to stimulated emission induced by the pump mode. The photons emitted this way are not included in the mean photon number for the cavity mode.

We next proceed to calculate the normally-ordered variance of the photon number for the cavity mode. The normally-ordered variance of the photon number is defined as [21]

$$
: \Delta n^{2}:=\left\langle: \hat{n}^{2}:\right\rangle-\langle\hat{n}\rangle^{2}
$$

With the aid of Eq. (72), the normally-ordered variance of the photon number can be put in the form

$$
: \Delta n^{2}:=\Delta n^{2}-\hat{n},
$$

with $\Delta n^{2}$ and $\bar{n}$ being the variance and the mean of the photon number for the cavity mode. Furthermore, the variance of the photon number can be expressed as

$$
\Delta n^{2}=\left\langle\hat{a}^{2} \hat{a}^{\dagger 2}\right\rangle-\bar{n}^{2}-3 \bar{n}-2 .
$$

Employing (73) one can write

$$
\left\langle\hat{a}^{2} \hat{a}^{\dagger 2}\right\rangle=\int d \alpha^{2} Q(\alpha) \alpha^{2} \alpha^{* 2} .
$$

Now applying the $Q$ function (69) in Eq. (80) and performing the integration, we obtain

$$
\left\langle\hat{a}^{2} \hat{a}^{\dagger 2}\right\rangle=E^{4}+4 E^{2} a-2 E^{2} b+2 a^{2}+b^{2},
$$


so that substitution of (75) and (81) into Eq. (79) results in

$$
\Delta n^{2}=2 E^{2} a+a^{2}+b^{2}-2 E^{2} b-E^{2}-a .
$$

On account of Eqs. (36), (67), and (68), the variance of the photon number at steady state turns out to be

$$
\Delta n_{s s}^{2}=\bar{n}_{s s}+\frac{4 \mu^{2}}{\lambda_{-}^{2}}\left(\frac{2 R-2 V+\varepsilon}{4 \lambda_{-}}\right)+2\left(\frac{2 R-2 V+\varepsilon}{4 \lambda_{-}}\right)^{2}+2\left(\frac{2 R+2 V-\varepsilon}{4 \lambda_{+}}\right)^{2} .
$$

Hence in view of this result, the normally-ordered variance of the photon number (77) goes over into

$$
: \Delta n_{s s}^{2}:=\frac{4 \mu^{2}}{\lambda_{-}^{2}}\left(\frac{2 R-2 V+\varepsilon}{4 \lambda_{-}}\right)+2\left(\frac{2 R-2 V+\varepsilon}{4 \lambda_{-}}\right)^{2}+2\left(\frac{2 R+2 V-\varepsilon}{4 \lambda_{+}}\right)^{2} .
$$

We see from Figure 7 that the normally-ordered variance of the photon number is positive. This indicates that the photon number statistics is super-Poissonian. In addition, we note that one effect of the coupling of the top and bottom levels is to decrease the normally-ordered variance of the photon number.

\subsection{Photon number distribution}

The photon number distribution for a single-mode light is expressible in terms of the $Q$ function as [3]

$$
P(n, t)=\frac{\pi}{n !} \frac{\partial^{2 n}}{\partial \alpha^{* n} \partial \alpha^{n}}\left[Q\left(\alpha^{*}, \alpha, t\right) e^{\alpha^{*} \alpha}\right]_{\alpha^{*}=\alpha=0} .
$$

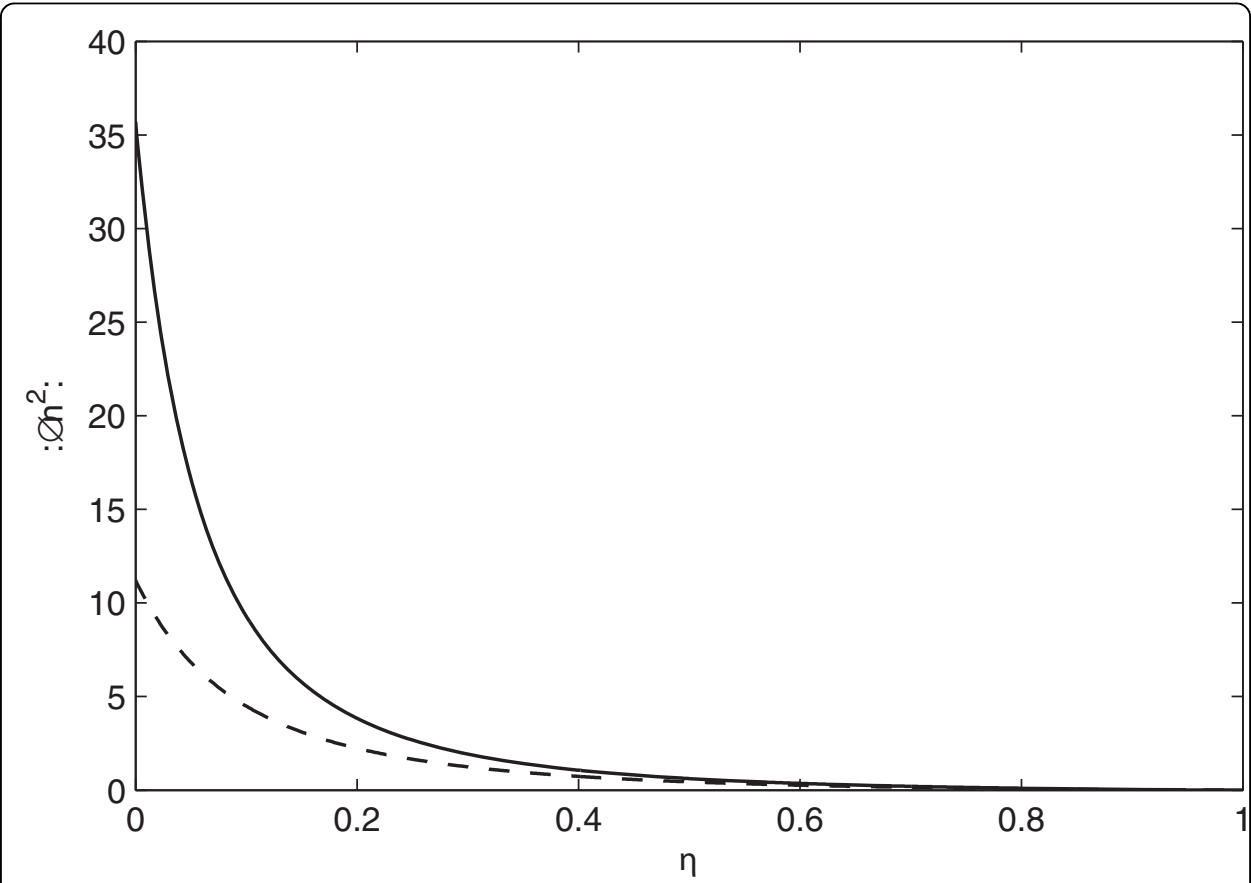

Figure 7 The normally-ordered variance of the photon number. Plots of the normally-ordered variance of the photon number [Eq. (84)] versus $\eta$ for $\varepsilon=3.5, \mu=5, A=100, \kappa=0.8$, and $\beta=0.2$ (solid curve) and $\beta=0.3$ (dotted curve). 
Now using the $Q$ function (69), the photon number distribution for the cavity mode under consideration is found to be

$$
\begin{aligned}
& P(n, t)=\left(c^{2}-d^{2}\right)^{1 / 2} e^{-(c+d) E^{2}} \\
& \sum_{i l m} \frac{n !(-1)^{l+m}(1-c)^{i}(c E+d E)^{2(n-i-l-m)} d^{l+m}}{2^{l+m} i ! l ! m !(n-i-2 l) !(n-i-2 m) !} .
\end{aligned}
$$

It is interesting to consider the special case in which the coherent driving light is absent $(\mu=0)$. Thus upon setting $\mu=0$ in Eq. (36), we get

$$
E=0 .
$$

With the aid of this, we find

$$
l=m
$$

and

$$
l=(n-i) / 2 .
$$

Hence on account of Eqs. (87) and (89) along with the fact that a factorial is defined for nonnegative integers, the photon number distribution can be put in the form [19]

$$
P(n, t)=\left(c^{2}-d^{2}\right)^{1 / 2} \sum_{l}^{[n]} \frac{n !(1-c)^{n-2 l} d^{2 l}}{2^{2 l}(n-2 l) ! l ! 2}
$$

where $[n]=\frac{n}{2}$ for even $n$ and $[n]=\frac{n-1}{2}$ for odd $n$. As can be seen from Figure 8 the steady-state photon number distribution decreases with the photon number. Though the photons are generated in pairs in this quantum optical system, there is a finite probability to find odd number of photons inside the cavity. This is due to the

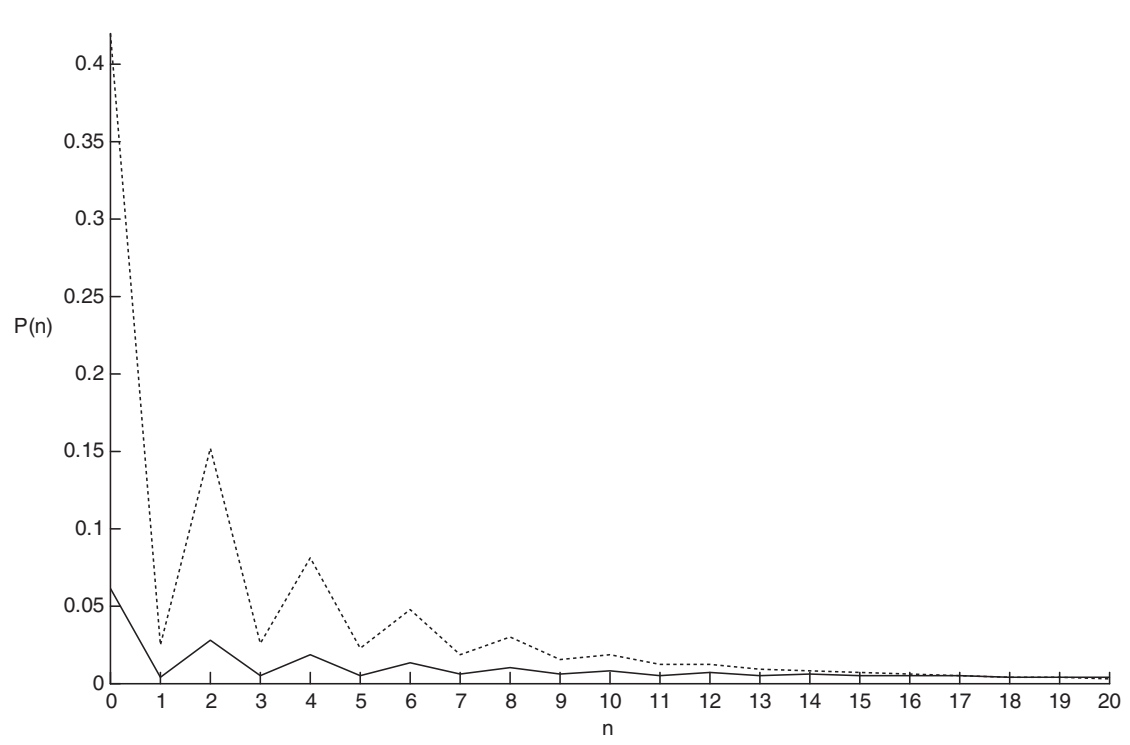

Figure 8 The photon number distribution. Plots of the photon number distribution [Eq. (90)] at steady state versus the photon number for $\beta=0, \eta=0.1, A=100, \kappa=0.8$, and $\varepsilon=5.3$ (solid curve) and $\varepsilon=0$ (dotted curve). 
damping of the cavity mode. In addition, the probability of finding even number of photons is in general greater than the probability of finding odd number of photons. One can also obtain using Eq. (90) that the probability of finding $n$ photons, with $n \leq$ 17 , is smaller for the light generated by the three-level laser with the parametric amplifier. And the opposite of this holds for $n>17$.

\section{Conclusions}

In this paper we have seen the simplicity with which the squeezing and statistical properties of the light, generated by a coherently driven degenerate three-level lasers whose cavity contains a parametric amplifier, could be analyzed with the aid of c-number Langevin equations. Applying the solutions of these equations, we have calculated the quadrature variance for the cavity and output modes. Our results show that the presence of the parametric amplifier increases the squeezing of the light generated by the system under consideration, while the driving light has no effect on the squeezing. Furthermore, it so happens that for small values of the amplitude of the pump mode, the coupling of the top and bottom levels enhances the degree of the intracavity squeezing significantly for $\eta=0$. Otherwise, it leads to a decrease in the intercavity squeezing.

It so turns out that for $\eta=0, A=100$, and $\kappa=0.8$ the maximum interacavity squeezing is $93 \%$ below the coherent-state level (occurs at $\beta=0.1$ ). This squeezing is exclusively due to the parametric amplifier and the coupling of the top and bottom levels. Furthermore, for $\eta=0.1$ and the above values of $A$ and $\kappa$ the maximum interacavity squeezing is found to be $94 \%$ below the coherent-state level (occurs at $\beta=0$ ). This squeezing is due to the parametric amplifier and the superposition of the top and bottom levels. In addition, we have shown that the cavity mode squeezing is greater than the output mode squeezing by $19 \%$. On the other hand, we have determined via the $Q$ function the mean and the normally-ordered variance of the photon number and the photon number distribution for the cavity mode. From the results we have found, we note that the driving coherent light and the parametric amplifier increase the mean of the photon number significantly. We have seen that one effect of the coupling of the top and bottom levels is to decrease the mean and normally-ordered variance of the photon number. This could be due to stimulated emission induced by the pump mode. The photons emitted this way do not contribute to the mean photon number of the cavity mode. Furthermore, we have also observed that the photon number statistics is super-Poissonian. In addition, we have found that there is a finite probability to find odd number of photons inside the cavity.

\section{Acknowledgements}

One of the authors, Tewodros, would like to thank the Abdus Salam ICTP for financial support.

Received: 1 January 2010 Accepted: 24 March 2010 Published: 24 March 2010

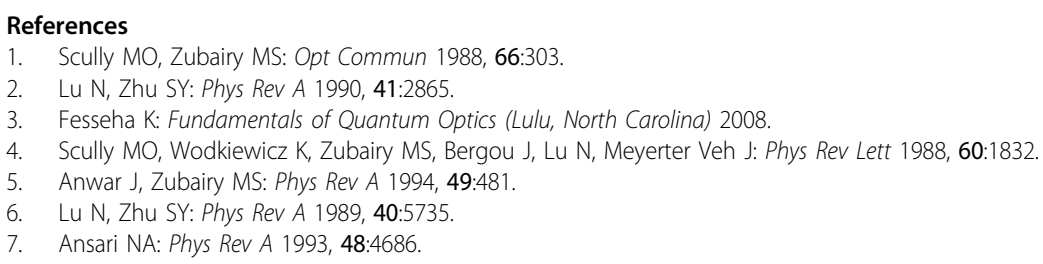


8. Tesfa S: J Phys B: At Mol Opt Phys 2008, 41:145501.

9. Ansari NA, Gea-Banacloche J, Zubairy MS: Phys Rev A 1990, 41:5179.

10. Anwar J, Zubairy MS: Phys Rev A 1992, 45:1804.

11. Fesseha K: Opt commun 1998, 156:145.

12. Agrawal GS, Adam G: Phys Rev A 1989, 39:6259.

13. Vyas R, Singh S: Phys Rev A 1989, 40:5147.

14. Daniel B, Fesseha K: Opt Commun 1998, 151:384.

15. Collet MJ, Gardiner CW: Phys Rev A 1984, 30:1386

16. Milburn GJ, Walls DF: Phys Rev A 1983, 27:392.

17. Wu LA, Xiao M, Kimble HJ: J Opt Soc Am B 1987, 4:1465.

18. Xiao M, Wu LA, Kimble HJ: Phys Rev Lett 1992, 59:278.

19. Fesseha K: Phys Rev A 2001, 63:033811.

20. Alebachew E, Fesseha K: Opt Commun 2006, 265:314.

21. Barnett SM, Radmore PM: Methods in Theoretical Quantum Optics Oxford University Press, New York 1997.

doi:10.1186/1754-0429-3-1

Cite this article as: Darge and Kassahun: Coherently driven degenerate three-level laser with parametric amplifier. PMC Physics B 2010 3:1.

Submit your next manuscript to BioMed Central and take full advantage of:

- Convenient online submission

- Thorough peer review

- No space constraints or color figure charges

- Immediate publication on acceptance

- Inclusion in PubMed, CAS, Scopus and Google Scholar

- Research which is freely available for redistribution 